\title{
Sensorineural Hearing Loss in Patients with Chronic Suppurative Otitis Media
}

\author{
E. S. Kolo • A. D. Salisu • A. M. Yaro • \\ O. G. B. Nwaorgu
}

Received: 17 June 2010/Accepted: 16 March 2011/Published online: 30 March 2011

(C) Association of Otolaryngologists of India 2011

\begin{abstract}
Even though there are other complications of chronic suppurative otitis media, the aspect of hearing loss is often studied. Nevertheless, the occurrence of sensorineural hearing loss in patients with this disease is still controversial. This study aim (1) to assess the association between sensorineural hearing loss and chronic suppurative otitis media, (2) to investigate some clinical factors that might affect the sensorineural component in patients with this disease. This was a retrospective analysis of the clinical records and pure tone audiograms of patients with chronic suppurative otitis media in a Nigerian Tertiary Health Institution from January 2003 to December 2008. Sixty-nine patients with a mean age of 28.93 years; standard deviation of 18.593 were studied. They had an age range of 4-73 years. The duration of otorrhea ranged from 2 months to 20 years; with a mean of 6.11 years (standard deviation of 6.393). The mean bone conduction threshold in the diseased ear was $39.07 \mathrm{~dB}$ (standard deviation of 12.028 ), and $10.26 \mathrm{~dB}$ (standard deviation of 2.620) in the control ear $(P<0.05)$. The mean bone conduction threshold differences between the diseased and control ears range from 21.69 to $34.52 \mathrm{~dB}$ across the frequencies 0.5 , 1.0, 2.0, and $4.0 \mathrm{kHz}$. These differences tended to increase with increasing frequency and were all significant $(P<0.05)$. In the diseased ears, there were no significant correlation between the age, duration of otorrhea, and the degree of SNHL $(r=0.186, \quad P>0.05 ; \quad r=0.190$, $P>0.05$ respectively). Patients with chronic suppurative otitis media had a significant degree of sensorineural
\end{abstract}

E. S. Kolo $(\bowtie)$ · A. D. Salisu · A. M. Yaro · O. G. B. Nwaorgu Department of Otorhinolaryngology, Aminu Kano Teaching Hospital, Kano, Nigeria

e-mail: emmyk90@yahoo.com hearing loss in this study. The higher frequencies were more affected; however, the patient's age and duration of otorrhea seem not to have any correlation with the degree of sensorineural hearing loss.

Keywords Chronic suppurative otitis media . Sensorineural hearing loss $\cdot$ Audiometry $\cdot$ Nigeria

\section{Introduction}

Chronic suppurative otitis media (CSOM) has assumed a world-wide importance. For instance, the United States government spends over 2 billion dollars yearly to treat acute and chronic ear infections [1]. In the developing countries, it has continued to constitute a heavy disease burden, with the prevalence of chronic ear infections being up to 72 cases per 1,000 inhabitants [2].

In spite of the fact that the complications of CSOM can be fatal [3, 4], hearing impairment is regarded the main health issue. Furthermore, the conductive hearing impairment resulting from this condition has been well acknowledged in the literature [5]. However, the relationship between sensorineural hearing loss (SNHL) and CSOM remains a controversial issue. For instance, some workers have consistently reported the presence of SNHL in patients with this condition [6-10]. On the contrary, some investigators have reiterated the fact that little or no such relationship exist $[11,12]$. Ironically, though the prevalence of this disease is still high in the developing countries; there is a dearth of information on its effect on hearing. The question is: what is the effect of this disease on the cochlear function of our patients? An analysis of this will help to bridge the gap in the current literature. 
Chronic suppurative otitis media is a persistent inflammation of the middle ear or mastoid cavity, and is characterised by recurrent or persistent ear discharge through a perforation of the tympanic membrane [13]. The treatment of this condition can be medical; with therapy directed at eradicating pathogenic aerobic and anaerobic organisms [14]. Those cases that are resistant to medical treatment might need surgical intervention. The objectives of this study were to assess the association between SNHL and $\mathrm{CSOM}$, and to investigate some clinical factors if any that might affect the sensorineural component in patients with this disease.

\section{Methods}

This was a retrospective analysis of the clinical records of patients with CSOM who attended the Otolaryngologic clinics of Aminu Kano Teaching Hospital (Northern Nigeria), over a 5-year period; from January 2003 to December 2008. The study was approved by the Institutional Review Board of the hospital.

The case notes of all patients with diagnosis of CSOM seen in the ENT out patient clinic were retrieved using their registration numbers in the ENT clinic register. Their respective pure tone audiograms were then retrieved. The Pure Tone Audiometry (PTA) was performed on dry ears in a sound proof booth by a trained and experienced audiologist; and with the same audiometer (calibrated Kamplex AD 229 with TDH 39P headphones). A 5 dB step procedure was employed, and a narrow band noise was used for masking.

Patients were recruited into the study if they met the following inclusion criteria: unilateral continuous otorrhea through a perforated tympanic membrane for at least 2 months, normal tympanic membrane in the contralateral ear based on otoscopy, no history of head trauma or meningitis, no previous tympanomastoid surgery, no systemic ototoxic drug therapy, no family history of congenital or acquired SNHL, and no post-traumatic tympanic membrane perforation. They were excluded if their clinical records were incomplete, or if they had no audiograms. The age, gender, duration of ear disease, and PTA results were all recorded.

\section{Data Analysis}

All the data obtained were entered into a specialized form designed for this study. They were analyzed using the Statistical Package for Social Sciences software version 13 (SPSS 13). The contralateral ear (paired) acted as a control. Bone conduction (BC) thresholds for the frequencies of
$0.5,1.0,2.0$, and $4.0 \mathrm{kHz}$ were considered for this study. In order to increase the sensitivity of the study, we defined SNHL as an intensity of $30 \mathrm{~dB}$ or more for the BC thresholds in two or more frequencies (those with significant cochlear damage) [6]. The main outcome measures were the $\mathrm{BC}$ threshold averages for the frequencies 0.5 , $1.0,2.0$, and $4.0 \mathrm{kHz}$; and with comparison between the control and diseased ears. The chi-square test was used to compare proportions and the differences between the diseased and control ears BC thresholds were analyzed with the Wilcoxon test. The association between age, duration of otorrhea, and degree of SNHL were tested by the Pearson correlation test. A $P$-value of $<0.05$ was considered statistically significant.

\section{Results}

A total of 106 CSOM patients' clinical notes were retrieved; however only 69 met the inclusion criteria and were analyzed. The clinical and demographic characteristics of these 69 patients are shown in Table 1.

The mean BC thresholds of the disease and control ears across the frequencies $(0.5,1,2$, and $4 \mathrm{kHz})$ are shown in Table 2.

The mean $\mathrm{BC}$ threshold in the diseased ear was $39.07 \mathrm{~dB}(\mathrm{SD}=12.028)$, and $10.26 \mathrm{~dB}(\mathrm{SD}=2.620)$ in the control ear $(P<0.05)$.

Table 3 shows the mean $\mathrm{BC}$ threshold differences between the diseased and control ears range from 21.69 to $34.52 \mathrm{~dB}$ across the four frequencies. These differences tended to increase with increasing frequency and were all significant $(P<0.05)$.

Table 1 Clinical and demographic characteristics of the study population

\begin{tabular}{lll}
\hline Characteristics & Number $(n)$ & Percentage $(\%)$ \\
\hline Sex & & 56.52 \\
Male & $39^{\mathrm{a}}$ & 43.48 \\
Female & $30^{\mathrm{b}}$ & \\
Age (years) & & \\
Range & $4-73$ & \\
Mean & $28.93(\mathrm{SD}=18.593)$ & \\
Duration of otorrhea & \\
Range & 2 months-20 years & \\
Mean & 6.11 years $(\mathrm{SD}=6.393)$ & 60.87 \\
Affected ear & & 39.13 \\
Right & 42 & \\
Left & 27 &
\end{tabular}

a,b No significant difference

$S D$ standard deviation 
Table 2 Comparison of mean bone conduction thresholds in the disease and control ears

\begin{tabular}{llr}
\hline Frequency $(\mathrm{kHz})$ & Mean BC in disease ear & Mean BC control ear \\
\hline 0.5 & $32.46(14.587)^{\mathrm{a}}$ & $10.14(4.470)$ \\
1 & $36.06(14.140)$ & $10.14(5.208)$ \\
2 & $42.18(15.183)$ & $9.97(4.585)$ \\
4 & $45.51(15.384)$ & $10.99(3.930)$ \\
\hline
\end{tabular}

${ }^{a}$ Standard deviation

Table 3 Mean bone conduction threshold differences between the disease and control ears

\begin{tabular}{llccc}
\hline $\mathrm{kHz}$ & 0.5 & 1 & 2 & 4 \\
$\mathrm{~dB}$ & $21.69(14.676)^{\mathrm{a}}$ & $25.66(15.183)$ & $32.28(16.309)$ & $34.63(15.144)$ \\
\hline
\end{tabular}

${ }^{\mathrm{a}}$ Standard deviation

Table 4 Mean air-bone gap in the diseased ears

\begin{tabular}{ll}
\hline Frequency $(\mathrm{kHz})$ & Mean air-bone gap $(\mathrm{dB})$ \\
\hline 0.5 & $40.48(26.311)^{\mathrm{a}}$ \\
1 & $36.43(19.567)$ \\
2 & $27.25(18.671)$ \\
4 & $28.50(17.925)$ \\
\hline
\end{tabular}

${ }^{a}$ Standard deviation

Table 4 shows the mean air-bone gap across the test frequencies in the diseased ears. These differences in mean were not statistically significant $(P>0.05)$.

In the diseased ears, there were no significant correlations between the age, duration of otorrhea, and the degree of SNHL $(r=0.186, P>0.05 ; r=0.190, P>0.05$ respectively). Also, there were no significant correlations between the air-bone gap and the degree of SNHL across the test frequencies in the diseased ears $(r=-0.279$, $P>0.05 ; r=-0.078, P>0.05 ; r=0.074, P>0.05$ and $r=-0.038, P>0.05$ for the frequencies $0.5,1,2$ and $4 \mathrm{kHz}$ respectively).

\section{Discussion}

Although numerous studies have documented the association between SNHL and CSOM, opinions still differ regarding the significance of this concept. In this study, we found a significant degree of SNHL amongst patients with CSOM-the mean BC thresholds across the speech frequencies were significantly higher in the diseased ears compared to the control ears. Also, the higher frequencies tend to be more affected. This is in support of the findings of MacAndie [7] and Redaelli et al. [15] in a similar study.
However, it is pertinent to note that the higher $\mathrm{BC}$ thresholds found in our study could possibly be because only patients with substantial cochlear damage (30 dB and above) were included in the analysis. In contrast, de Azevedo et al. [6] found no strong evidence of the effect of the disease on BC thresholds at any frequency.

This study found in the diseased ears, and across the test frequencies, that there were no significant differences in the mean air-bone gap. Also, there were no significant correlations between the air-bone gap and the degree of SNHL. On the contrary, though the Carhart's effect is most commonly found in otosclerosis, it has been well demonstrated in chronic otitis media among other middle ear conditions [16]. Therefore, it might be expected that there might be a worsening in the bone-conduction, and possibly a significantly decreased air-bone gap at 1 or $2 \mathrm{kHz}$.

Furthermore, this study found no significant correlation in the diseased ears between the degree of SNHL and the duration of otorrhea. Likewise, previous authors had also arrived at the same conclusion [6, 7]. Moreover, when we analyzed the effect of the patients' age on the degree of SNHL in the diseased ears, there was still no significant correlation. On the contrary, other workers had found that increasing age was a risk factor in the evolution of SNHL in patients with CSOM $[6,15]$. The discrepancies in the study populations with respect to the patients' ages could possibly be responsible for the differences in our findings.

Therefore, the findings from this study lend credence to the theory that cochlear damage in patients with CSOM might be due to bacterial toxins that diffuse through the round window membrane. Subsequently, these toxins might cause damage to the hair cells especially those at the cochlear base; where the hair cells are sensitive to high frequency sounds $[17,18]$. However, we acknowledge that this study lack control for the possible effect of a confounding factor - the use of topical antibiotics. This was a limitation as almost all our patients had admitted to have used such medications. But quite interestingly, some investigators have found little or no evidence that topical antibiotics can cause significant SNHL in humans [19].

\section{Conclusion}

A significant degree of SNHL in patients with CSOM was noted in this study. The higher frequencies were more affected, and there were no correlations between the degree of SNHL and the patients' age and duration of otorrhea.

Acknowledgments We wish to thank the entire staff of ENT department of Aminu Kano Teaching Hospital for their assistance during the course of this work. We also thank the departmental secretary $\mathrm{Mr}$. Abdullahi for helping in typing the manuscripts. 


\section{References}

1. Bluestone CD (1981) Recent advances in the pathogenesis, diagnosis, and management of otitis media. Pediatr Clin N Am 28(4):727-755

2. Ologe FE, Nwawolo CC (2002) Prevalence of chronic suppurative otitis media (CSOM) among school children in a rural community in Nigeria. Niger Postgrad Med J 9(2):63-66

3. Miura MS, Krumennauer RC, Lubianca Neto JF (2005) Intracranial complications of chronic suppurative otitis media in children. Braz J Otorhinolaryngol 71(5):639-643

4. Mostafa BE, El Fiky LM, El Sharnouby MM (2009) Complications of suppurative otitis media: still a problem in the $21 \mathrm{st}$ century. ORL J Otorhinolaryngol Relat Spec 71(2):87-92

5. Mills RP (1997) Management of chronic suppurative otitis media. In: Kerr AG, Booth JB (eds) Scott-Brown's otolaryngology, vol. 3, 6th edn. Butterwoth-Heinemann, Oxford, p 3/10/4

6. de Azevedo AF, Pinto DCG, Alves de Souza NJ, Greco DB, Goncalves DU (2007) Sensorineural hearing loss in chronic suppurative otitis media with and without cholesteatoma. Braz J Otorhinolaryngol 73(5):671-674

7. MacAndie C, O'Reilly BF (1999) Sensorineural hearing loss in chronic otitis media. Clin Otolaryngol Allied Sci 24(3):220-222

8. da Costa SS, Rosito LP, Dornelles C (2009) Sensorineural hearing loss in patients with chronic otitis media. Eur Arch Otorhinolaryngol 266(2):221-224

9. Lasisi AO, Sulaiman OA, Afolabi OA (2007) Socio-economic status and hearing loss in chronic suppurative otitis media in Nigeria. Ann Trop Paediatr 27(4):291-296

10. Feng H, Chen Y (2004) Analysis of sensorineural hearing loss in chronic suppurative otitis media. Lin Chuang Er Bi Yan Hou Ke Za Zhi 18(10):579-581
11. Kaplan DM, Fliss DM, Kraus M, Dagan R, Leiberman A (1996) Audiometric findings in children with chronic suppurative otitis media without cholesteatoma. Int $\mathrm{J}$ Pediatr Otorhinolaryngol 35(2):89-96

12. Noordzij JP, Dodson EE, Ruth RA, Arts HA, Lambert PR (1995) Chronic otitis media and sensorineural hearing loss: is there a clinically significant relation? Am J Otol 16(4):420-423

13. Acuin J (2002) Extracts from "Concise clinical evidence": chronic suppurative otitis media. BMJ 325(7373):1159-1160

14. Brook I (2008) The role of anaerobic bacteria in chronic suppurative otitis media in children: implications for medical therapy. Anaerobe 14(6):297-300

15. Redaelli de Zinis LO, Campovecchi C, Parrinello G, Antonelli AR (2005) Predisposing factors for inner ear hearing loss association with chronic otitis media. Int J Audiol 44(10):593-598

16. Yasan H (2007) Predictive role of Carhart's notch in pre-operative assessment for middle-ear surgery. J Laryngol Otol 121(3): 219-221

17. Spandow O, Anniko M, Hellstrom S (1989) Inner ear disturbances following inoculation of endotoxin into the middle ear. Acta Otolaryngol 107(1-2):90-96

18. Goycoolea MV, Paparella MM, Juhn SK, Carpenter AM (1980) Oval and round window changes in otitis media. Potential pathways between middle ear and inner ear. Laryngoscope 90: 1387-1391

19. Browning GG, Gatehouse S, Calder IT (1988) Medical management of active chronic otitis media: a controlled study. J Laryngol Otol 102(6):491-495 